\title{
The intersection power graph associated with a finite group
}

\author{
Wei Lv, Xuanlong Ma* \\ School of Science, Xi'an Shiyou University, Xi'an 710065 China \\ *Corresponding author, e-mail: xuanlma@mail.bnu.edu.cn
}

Received 30 Sep 2020

Accepted 8 Aug 2021

\begin{abstract}
We characterize the independence number of the intersection power graph of a group, which extends some results in Aprose et al [Punjab Univ J Math 52 (2020):47-53]. Moreover, the finite groups with dominatable intersection power graphs are characterized, which generalizes some results in Bera [Electron J Graph Theory Appl 6 (2018):178-189]. Furthermore, we characterize the finite groups whose intersection power graphs equal to their power graphs, enhanced power graphs, commuting graphs, and order supergraphs.
\end{abstract}

KEYWORDS: intersection power graph, power graph, enhanced power graph, finite group

MSC2010: 05C25

\section{INTRODUCTION}

A simple graph is an undirected graph without loops and multiple edges. All graphs considered in this paper are finite and simple. By associating a graph to an algebraic structure, the investigation of the interplay between the algebraic structure and graph theory has been actively investigated in the literature. For example, the Cayley graph of a group, which has a long history.

Let $G$ be a group. The undirected power graph of $G$, denoted $\mathscr{P}(G)$, is a graph whose vertex set is $G$ and two distinct vertices are adjacent if one is a power of the other. Kelarev and Quinn [1] first introduced the concept of the power graph of a group, which is a directed graph. The concept of the undirected power graph of a group was introduced first by Chakrabarty et al [2]. For convenience, in this paper, we always use "power graph" to denote an undirected power graph. The subgraph of $\mathscr{P}(G)$ induced by $G \backslash\{e\}$ is called the proper power graph of $G$ and is denoted by $\mathscr{P}^{*}(G)$. In past two decades, the study of the (proper) power graph of a group has been growing. See, for example, [3-6] and the survey paper [7] with many results and open questions on the power graph of a group.

The commuting graph of $G$, denoted $\mathscr{C}(G)$, is a graph whose vertices are the elements of $G$ and two distinct vertices $x, y$ are adjacent if $x y=y x$. In order to classify finite simple groups, Brauer and Fowler [8] first studied the graph. Notice that every element of the center of $G$ is adjacent to any other vertex. As a result, the vertex set of the commuting graph of $G$ is assumed to the set of all non-central elements of $G$. For some results on commuting graphs, see $[9,10]$ and references therein. The enhanced power graph of $G$, denoted $\mathscr{P}_{E}(G)$, is the graph whose vertex set is $G$, and two distinct vertices are adjacent if they can generate a cyclic subgroup of $G$. Clearly, $\mathscr{P}(G)$ is a spanning subgraph of $\mathscr{P}_{E}(G)$. To measure how close power graphs of groups is to commuting graphs of groups, Aalipour et al [11] first introduced the concept of an enhanced power graph of a group. For some more properties on the enhanced power graph of a group, see [12]. The order supergraph of $\mathscr{P}(G)$, denoted $\mathscr{S}(G)$, is a graph whose vertex set is $G$ and two distinct elements $x, y \in G$ are adjacent if $o(x) \mid o(y)$ or $o(y) \mid o(x)$. For convenience, we also call $\mathscr{S}(G)$ as the order supergraph of $G$. In 2017, Hamzeh and Ashrafi [13] first introduced the order supergraph and studied its full automorphism group. In fact, the authors in [13] called $\mathscr{S}(G)$ as the main supergraph of $\mathscr{P}(G)$. In 2018, Hamzeh and Ashrafi [14] obtained some more properties of $\mathscr{S}(G)$.

As stated in [15], one can define a graph over a group by using a poset consisting of all cyclic subgroups of the group, and use Hasse diagram of the poset to visualize some algebraic properties of the group. Motivated by this, Bera [15] defined the intersection power graph $\mathscr{P}_{I}(G)$ of a group $G$, where the vertex set of $\mathscr{P}_{I}(G)$ is $G$, and distinct vertices $x$ and $y$ are adjacent if either one of $\{x, y\}$ is the 
identity element of $G$, or $\langle x\rangle \cap\langle y\rangle$ is non-trivial. In [15], the author studied some properties of $\mathscr{P}_{I}(G)$ and determined the full automorphism group of the intersection power graph of a cyclic group.

Every group considered in this paper is finite. In general, $G$ always denotes a group and its identity element is $e$. Let $g \in G$, the order of $g$, denoted $o(g)$, is the cardinality of the cyclic subgroup $\langle g\rangle$ generated by $g$. If $M$ is a cyclic subgroup of $G$ and is not a proper subgroup of some cyclic subgroup of $G$, then $M$ is called a maximal cyclic subgroup of $G$. The set of maximal cyclic subgroups of $G$ is denoted by $\mathscr{M}_{G}$. We remark that the size of $\mathscr{M}_{G}$ is 1 if and only if $G$ is a cyclic group. Also, $\mathbb{Z}_{n}$ denotes the cyclic group of order $n$. Let $\Gamma$ is a graph. $\Gamma$ is called complete if every two distinct vertices of $\Gamma$ is connected by an edge. The independence number of $\Gamma$, denoted $\alpha(\Gamma)$, is defined as the maximum cardinality of a set of pairwise non-adjacent vertices which is called an independent set of $\Gamma$. An independent set is called maximal in $\Gamma$ if no vertex can be added without violating independence. A vertex of $\Gamma$ is called a dominating vertex if it is adjacent to every other vertex of $\Gamma$. Note that $e$ is adjacent to every other vertex in $\mathscr{P}_{I}(G)$. Therefore, $e$ is a dominating vertex in $\mathscr{P}_{I}(G)$. An intersection power graph $\mathscr{P}_{I}(G)$ is called dominatable if $G$ has a non-trivial dominating vertex.

In this paper, we first characterize the independence number of the intersection power graph of a group (see Theorem 2), which implies Theorems 4.1 and 4.2 of [16]. We then characterize all groups whose intersection power graphs are dominatable (see Theorem 3), which generalizes Theorems 4.1 and 4.3 of [15]. There are various graphs defined on the set of elements of a group. It is natural to consider the following question.

Question 1 For which groups is the intersection power graph equal to the power graph, the enhanced power graph, the commuting graph, or the order supergraph?

Motivated by this question, we finally characterize the groups whose intersection power graphs equal to the power graphs, the enhanced power graphs, the commuting graphs, and the order supergraphs.

\section{INDEPENDENCE NUMBER}

In this section, we characterize the independence number of the intersection power graph of a group. Our main result is Theorem 2, which implies Theorems 4.1 and 4.2 of [16].
Theorem $2 \alpha\left(\mathscr{P}_{I}(G)\right)$ is the size of the set of all subgroups of prime order of $G$.

Proof: Let $\mathscr{N}_{G}$ be the set of all subgroups of prime order in $G$. Write

$$
\mathscr{N}_{G}=\left\{\left\langle u_{1}\right\rangle,\left\langle u_{2}\right\rangle, \ldots,\left\langle u_{t}\right\rangle\right\}
$$

Let $S$ be an independent set of $\mathscr{P}_{I}(G)$ such that every element of $S$ has prime order. we first claim $|S| \leqslant t$. Assume, to the contrary, that $|S|>t$. Then by (1), there exist distinct $x, y \in S$ such that $\langle x\rangle=\langle y\rangle$. It follows that $\langle x\rangle \cap\langle y\rangle \neq\{e\}$. Thus, $x$ and $y$ are adjacent in $\mathscr{P}_{I}(G)$, this contradicts that $S$ is an independent set. Therefore, our claim is valid.

Now let $X=\left\{x_{1}, x_{2}, \ldots, x_{k}\right\}$ be an independent set with $\alpha\left(\mathscr{P}_{I}(G)\right)=k$. In the following, we prove $k \leqslant t$. If $o\left(x_{i}\right)$ is a prime for each $i \in\{1,2, \ldots, k\}$, then the claim as above implies $k \leqslant t$, as desired. Thus, we may assume that there exists $x_{j} \in X$ such that $o\left(x_{j}\right)$ is not a prime. In the following, we prove that $o\left(x_{j}\right)$ is a prime power. Suppose for a contradiction that $o\left(x_{j}\right)$ has two distinct prime divisors $p, q$. Let $u, v \in\left\langle x_{j}\right\rangle$ with $o(u)=p$ and $o(v)=q$. Notice that $X$ is an independent set. Clearly, $u, v \notin X$. If there exists a vertex in $X \backslash\left\{x_{j}\right\}$ such that it is adjacent to $u$ or $v$, then the vertex must be adjacent to $x_{j}$, a contradiction. It follows that $\{u, v\} \cup\left(X \backslash\left\{x_{j}\right\}\right)$ is an independent set of size $k+1$, this contradicts $\alpha\left(\mathscr{P}_{I}(G)\right)=k$. We conclude that the order of any element of $X$ is either a prime or a prime power. Without loss of generality, now let $\left\{x_{1}, x_{2}, \ldots, x_{l}\right\}$ be the set of all non-prime-order elements of $X$, where $l \leqslant k$. For each $i, 1 \leqslant i \leqslant l$, take $y_{i} \in\left\langle x_{i}\right\rangle$ such that $o\left(y_{i}\right)$ is a prime. Then it is easy to see that

$$
Y=\left\{y_{1}, y_{2}, \ldots, y_{l}, x_{l+1}, \ldots, x_{k}\right\}
$$

is an independent set of size $k$. Note that every element of $Y$ has prime order. Thus, by the claim as above, we have $k \leqslant t$, as desired. We conclude $\alpha\left(\mathscr{P}_{I}(G)\right) \leqslant t$.

Let $N=\left\{u_{1}, u_{2}, \ldots, u_{t}\right\}$. Clearly, $N$ is an independent set of $\mathscr{P}_{I}(G)$. It follows that $\alpha\left(\mathscr{P}_{I}(G)\right) \geqslant t$. This implies that $\alpha\left(\mathscr{P}_{I}(G)\right)=t=\left|\mathscr{N}_{G}\right|$.

For $n \geqslant 3$, the dihedral group of order $2 n$, denoted $D_{2 n}$, is presented by

$$
D_{2 n}=\left\langle a, b: a^{n}=b^{2}=e, b a b=a^{-1}\right\rangle .
$$

It is easy to see that

$$
\left\{\begin{aligned}
D_{2 n} & =\langle a\rangle \cup\left\{b, a b, a^{2} b, \ldots, a^{n-1} b\right\}, \\
o\left(a^{i} b\right) & =2 \text { for any } i \leqslant 1 \leqslant n,
\end{aligned}\right.
$$




$$
\mathscr{M}_{D_{2 n}}=\left\{\langle a\rangle,\langle a b\rangle,\left\langle a^{2} b\right\rangle, \ldots,\left\langle a^{n} b\right\rangle\right\}
$$

and

$$
\left\{\begin{array}{l}
Z\left(D_{2 n}\right)=\{e\} \text { if } n \text { is odd; } \\
Z\left(D_{2 n}\right)=\left\{e, a^{n / 2}\right\} \text { if } n \text { is even, }
\end{array}\right.
$$

where $Z\left(D_{2 n}\right)$ is the center of $D_{2 n}$. For $m \geqslant 2$, the generalized quaternion group, denoted $Q_{4 m}$, is presented by

$$
Q_{4 m}=\left\langle x, y: x^{m}=y^{2}, x^{2 m}=e, y^{-1} x y=x^{-1}\right\rangle .
$$

For more information see [17]. We remark that $Q_{4 m}$ has order $4 m$,

$$
o\left(x^{m}\right)=2, \quad o\left(x^{i} y\right)=4, \text { for any } i \leqslant 1 \leqslant m
$$

and

$\mathscr{M}_{Q_{4 m}}=\left\{\langle x\rangle,\langle x y\rangle,\left\langle x^{2} y\right\rangle, \ldots,\left\langle x^{m} y\right\rangle\right\}, x^{m} \in \bigcap_{M \in \mathscr{M}_{Q_{4 m}}} M$.

Clearly, $x^{m} \in Z\left(Q_{4 m}\right)$.

For a positive integer $n$, the set of all prime factors of $n$ is denoted by $\pi(n)$. Also, denote by $\mathbb{Z}_{p}^{k}$ the elementary abelian $p$-group of order $p^{k}$, where $p$ is a prime and $k$ is a positive integer. Notice that $\mathbb{Z}_{p}^{k}$ has $\frac{p^{k}-1}{p-1}$ maximal cyclic subgroups and every maximal cyclic subgroup is isomorphic to $\mathbb{Z}_{p}$.

Combining (2), (3), (5), (6), and Theorem 2, we have the following examples.

\section{Example 1}

(i) Let $n \geqslant 2$. Then $\alpha\left(\mathscr{P}_{I}\left(\mathbb{Z}_{n}\right)\right)=\pi(n)$.

(ii) Let $k \geqslant 2$ and $p$ a prime. Then

$$
\alpha\left(\mathscr{P}_{I}\left(\mathbb{Z}_{p}^{k}\right)\right)=\frac{p^{k}-1}{p-1} .
$$

(iii) $\alpha\left(\mathscr{P}_{I}\left(D_{2 n}\right)\right)=\pi(n)+n$.

(iv) $\alpha\left(\mathscr{P}_{I}\left(Q_{4 m}\right)\right)=\pi(2 m)$.

Recall now an elementary result on $p$-groups.

Lemma 1 (Theorem 5.4.10 [18]) Given a prime $p$, a p-group having a unique subgroup of order $p$ is either generalized quaternion or cyclic.

Note that $\mathscr{P}_{I}(G)$ is complete if and only if $\alpha\left(\mathscr{P}_{I}(G)\right)=1$. In the following, we classify all groups whose intersection power graphs are complete. By an alternative method, Bera [15] also obtained the result (see Theorem 3.1 [15]).

Corollary $1 \mathscr{P}_{I}(G)$ is complete if and only if $G$ is either a cyclic p-group or a generalized quaternion 2group.
Proof: Since both a cyclic $p$-group and a generalized quaternion 2-group have a unique subgroup of prime order, their intersection power graphs have independence number 1 by Theorem 2. Conversely, suppose that $\alpha\left(\mathscr{P}_{I}(G)\right)=1$. Then Theorem 2 implies $G$ has a unique subgroup of prime order. As a result, $G$ must be a $p$-group. Now the desired result follows from Lemma 1.

\section{DOMINATABILITY}

In this section, we characterize all the groups whose intersection power graphs are dominatable (see Theorem 3), which also generalizes Theorems 4.1 and 4.3 of [15].

In the following, we use $\Psi$ to denote the set of all groups $G$ such that the following two conditions hold:

(i) For any prime divisor $p$ of $|G|, G$ has a unique subgroup of order $p$.

(ii) If $p_{1}, p_{2}, \ldots, p_{t}$ are all prime divisor of $|G|$, then $G$ has a cyclic subgroup of order $\prod_{i=1}^{t} p_{i}$.

\section{Example 2}

(i) Every cyclic group belongs to $\Psi$.

(ii) Let $m \geqslant 2$. Then the generalized quaternion group $Q_{4 m}$ belongs to $\Psi$.

(iii) If $G$ is the semidirect product of $\mathbb{Z}_{5}$ and $\mathbb{Z}_{8}$ via square map, that is

$$
G=\left\langle a, b: a^{5}=b^{8}=e, b a b^{-1}=a^{2}\right\rangle,
$$

then $G \in \Psi$.

(iv) If $G$ is the semidirect product of $\mathbb{Z}_{5}$ and $\mathbb{Z}_{16}$ via inverse map, that is

$$
G=\left\langle a, b: a^{5}=b^{16}=e, b a b^{-1}=a^{-1}\right\rangle,
$$

then $G \in \Psi$.

Now we state our main theorem of the section.

Theorem $3 \mathscr{P}_{I}(G)$ is dominatable if and only if $G \in \Psi$.

Proof: We first prove the sufficiency. Suppose that $G \in \Psi$. Let $p_{1}, p_{2}, \ldots, p_{t}$ be all prime divisor of $|G|$. Then $G$ has a cyclic subgroup of order $\prod_{i=1}^{t} p_{i}$, say, $\langle x\rangle$. Let $y \in G \backslash\{x, e\}$. Taking $\langle z\rangle \subseteq\langle y\rangle$ such that $o(z)=p_{i}$ for some $1 \leqslant i \leqslant t$, we deduce that $\langle z\rangle \subseteq\langle x\rangle \cap\langle y\rangle$ by (i). It follows that $x$ is adjacent to $y$ in $\mathscr{P}_{I}(G)$. Since $x$ is adjacent to $e$ in $\mathscr{P}_{I}(G), x$ is a dominating vertex, which implies that $\mathscr{P}_{I}(G)$ is dominatable.

We next prove the necessity. Suppose that $\mathscr{P}_{I}(G)$ is dominatable. Let $a$ be a dominating vertex 
of $\mathscr{P}_{I}(G)$, and let $p_{1}, p_{2}, \ldots, p_{t}$ be all prime divisor of $|G|$. Suppose for a contradiction that $G$ has two distinct subgroups of order $p_{i}$ where $1 \leqslant i \leqslant t$, say, $\langle b\rangle$ and $\langle c\rangle$. Because $o(b)=o(c)=p_{i}$, where $p_{i}$ is a prime, it follows that $\langle b\rangle,\langle c\rangle \leqslant\langle a\rangle$, which is in contradiction with $\langle b\rangle \neq\langle c\rangle$. As a result, $G$ has a unique subgroup of order $p_{i}$ for each $1 \leqslant i \leqslant t$.

Now let $\left\langle b_{i}\right\rangle$ be the unique subgroup of order $p_{i}$ for each $1 \leqslant i \leqslant t$. If $t=1$, then clearly, $G$ has a cyclic subgroup $\left\langle b_{1}\right\rangle$ of order $p_{1}$, which implies $G \in \Psi$, as desired. Thus, we now may assume that $t \geqslant 2$. Note that $b_{i}$ is adjacent to $a$ for each $1 \leqslant$ $i \leqslant t$. It follows that $\left\langle b_{i}\right\rangle \cap\langle a\rangle=\left\langle b_{i}\right\rangle$, and so $b_{i} \in$ $\langle a\rangle$ for each $1 \leqslant i \leqslant t$. This means that every of $p_{1}, p_{2}, \ldots, p_{t}$ is a prime divisor of $|\langle a\rangle|$, that is, $\langle a\rangle$ has a cyclic subgroup of order $\prod_{i=1}^{t} p_{i}$. Thus, we have $G \in \Psi$.

Corollary 2 Let $G$ be a group such that $\mathscr{G}_{I}(G)$ is dominatable, and let a be a non-trivial element of $G$. Then the closed neighbourhood of a in $\mathscr{P}_{I}(G)$ is $G$ if and only if $o(a)$ is divisible by every prime divisor of $|G|$.

Note that a nilpotent group is a direct product of its Sylow subgroups. Combining Theorem 3 and Lemma 1, we have the following, where Corollary 3 (i) extends Theorems 4.1 and 4.3 of [15].

\section{Corollary 3}

(i) Let $G$ be a nilpotent group. Then $\mathscr{P}_{I}(G)$ is dominatable if and only if $G$ is isomorphic to either a cyclic group or $Q_{2^{n}} \times\langle g\rangle$, where $n \geqslant 3$ and $o(g)$ has odd order.

(ii) Let $m \geqslant 2$. Then $Q_{4 m}$ is dominatable.

(iii) Let $n \geqslant 3$. Then $D_{2 n}$ is not dominatable.

\section{COMPARING TO THE INTERSECTION POWER GRAPH AND THE OTHERS}

There are various graphs defined on the set of elements of a group. For example, in introduction of the paper, we mention the power graph, the enhanced power graph, the commuting graph, and the order supergraph of a group. Motivated by Question 1, in this section, we characterize the groups whose intersection power graphs equal to their power graphs, enhanced power graphs, commuting graphs, and order supergraphs.

\section{Intersection power graph and power graph}

The following result follows directly from the definitions of intersection power graph and power graph.
Observation 4 For a group $G$, the intersection power graph of $G$ is equal to its power graph if and only if $G$ satisfies the following property: for any $x, y \in G$, $\langle x\rangle \cap\langle y\rangle \neq\{e\}$ implies that $\langle x\rangle \subseteq\langle y\rangle$ or $\langle y\rangle \subseteq\langle x\rangle$.

A group is called a P-group [19] if every nontrivial element of the group has prime order. For example, both the dihedral group of order 6 and the alternating group on 5 letters are $P$-groups.

Example 3 If $G$ is a $P$-group, then the intersection power graph of $G$ is equal to its power graph.

Observation 4 implies the following corollary, which gives a necessary condition for $\mathscr{P}_{I}(G)=$ $\mathscr{P}(G)$.

Corollary 4 If $\mathscr{P}_{I}(G)=\mathscr{P}(G)$, then every two distinct maximal cyclic subgroups of $G$ has trivial intersection.

Remark that the converse of Corollary 4 is not true. In fact, by (3), it is easy to see that $D_{24}$ and $D_{60}$ are two counterexamples.

Lemma 2 Suppose that $G$ is a group with $\mathscr{P}_{I}(G)=$ $\mathscr{P}(G)$. Then

(i) G has no subgroup $\mathbb{Z}_{p} \times \mathbb{Z}_{p q}$, where $p$ and $q$ are primes;

(ii) G has no element of order $p^{2} q$, where $p$ and $q$ are distinct primes;

(iii) G has no element of order pqr, where $p, q$ and $r$ are distinct primes;

(iv) $G$ has no subgroup isomorphic to a generalized quaternion group.

Proof: (i) Suppose for a contradiction that $\mathbb{Z}_{p} \times \mathbb{Z}_{p q}$ is a subgroup of $G$, where $p$ and $q$ are primes. Then $(0, p) \in\langle(1,1)\rangle \cap\langle(0,1)\rangle$. However, $\langle(1,1)\rangle \nsubseteq$ $\langle(0,1)\rangle$ and $\langle(0,1)\rangle \nsubseteq\langle(1,1)\rangle$ since $o((1,1))=$ $o((0,1))=p q$, contrary to Observation 4 .

(ii) Suppose for a contradiction that $G$ has an element $x$ of order $p^{2} q$, where $p$ and $q$ are distinct primes. Then in $\langle x\rangle$, we have $x^{p q} \in\left\langle x^{p}\right\rangle \cap\left\langle x^{q}\right\rangle$. Since $o\left(x^{p}\right)=p q$ and $o\left(x^{q}\right)=p^{2}$, it follows that $\left\langle x^{p}\right\rangle \nsubseteq\left\langle x^{q}\right\rangle$ and $\left\langle x^{q}\right\rangle \nsubseteq\left\langle x^{p}\right\rangle$, contrary to Observation 4 .

(iii) Suppose for a contradiction that $G$ has an element $x$ of order $p q r$, where $p, q$ and $r$ are distinct primes. We deduce $x^{p q} \in\left\langle x^{p}\right\rangle \cap\left\langle x^{q}\right\rangle$. Notice that $o\left(x^{p}\right)=q r$ and $o\left(x^{q}\right)=p r$. We have $\left\langle x^{p}\right\rangle \nsubseteq\left\langle x^{q}\right\rangle$ and $\left\langle x^{q}\right\rangle \nsubseteq\left\langle x^{p}\right\rangle$, contrary to Observation 4 .

(iv) Suppose for a contradiction that $G$ has a subgroup $H$ which is a generalized quaternion 
group. By (5) and (6), there exist two distinct cyclic subgroups of order 4 in $H$ such that their intersection has size 2, contrary to Observation 4.

In the following, we use $\Phi$ to denote the set of all finite groups $G$ such that the following two conditions hold:

(i) $G$ is a non-cyclic $p$-group, where $p$ is a prime.

(ii) $G$ has a maximal cyclic subgroup $\langle x\rangle$, and the order of every element in $G \backslash\langle x\rangle$ is $p$.

In other words, if $G$ has a maximal cyclic subgroup of order at least $p^{2}$, then $G$ has precisely one maximal cyclic subgroup of order at least $p^{2}$.

Remark $1 G \in \Phi$ if $G$ is one of the following:

(a) The elementary abelian $p$-group $\mathbb{Z}_{p}^{m}$ for some prime $p$ and positive integer $m$;

(b) For any odd prime $q$, the group $U T(3, q)$ is defined as the unitriangular matrix group of degree 3 over the prime field $\mathbb{F}_{q}$. Explicitly,

$$
U T(3, p)=\left\{\left(\begin{array}{ccc}
0 & a_{12} & a_{13} \\
0 & 1 & a_{23} \\
0 & 0 & 1
\end{array}\right): a_{12}, a_{13}, a_{23} \in \mathbb{F}_{q}\right\}
$$

and its operation is the usual matrix multiplication. In fact, $U T(3, q)$ is the unique non-abelian group of order $q^{3}$ and exponent $q$;

(c) The dihedral group $D_{2 \cdot 2^{m}}$ for some positive integer $m \geqslant 2$.

Lemma 3 (Theorem $2.12[2]) \mathscr{P}(G)$ is complete if and only if $G$ is a cyclic group of prime power order.

Theorem 5 Let $G$ be a nilpotent group. $\mathscr{P}_{I}(G)=$ $\mathscr{P}(G)$ if and only if one of the following holds:

(i) $G$ is a cyclic p-group;

(ii) $G$ is a cyclic group of order $p q$, where $p$ and $q$ are two distinct primes;

(iii) $G \in \Phi$.

Proof: We first prove the sufficiency. If $G$ is a cyclic $p$-group, then by Corollary 1 and Lemma 3, we have $\mathscr{P}_{I}(G)=\mathscr{P}(G)$. If $G$ is a group of (ii) and (iii), then by Observation 4 , one can obtain easily that $\mathscr{P}_{I}(G)=$ $\mathscr{P}(G)$.

We next prove the necessity. Suppose that $\mathscr{P}_{I}(G)=\mathscr{P}(G)$. Assume that $G$ is not a $p$-group. Note that a nilpotent group is a direct product of its Sylow subgroups. By Lemma 2 (iii), we deduce that $|G|$ has precisely two distinct prime divisors, say, $p$ and $q$ with $p<q$. Let $\langle x\rangle$ and $\langle y\rangle$ be two cyclic subgroups of order $p$ and $q$, respectively. If $G$ has a cyclic subgroup $\langle z\rangle$ of order $q$ with $\langle z\rangle \neq\langle y\rangle$, then $\langle x y\rangle \cap\langle x z\rangle=\langle x\rangle$ and $o(x y)=o(x z)=p q$, a contradiction by Observation 4 . Thus, we conclude that $G$ has a unique cyclic subgroup $\langle y\rangle$ of order $q$. Similarly, we have that $G$ has a unique cyclic subgroup $\langle x\rangle$ of order $p$. If $p>2$, then by Lemma 1 , every Sylow subgroup of $G$ is cyclic, and so $G$ is a cyclic group of order $p q$ from Lemma 2 (ii), as desired. Similarly, if $p=2$, it follows from Lemma 1 , Lemma 2 (ii) and Lemma 2 (iv) that $G$ is a cyclic group of order $p q$, as desired.

Assume now that $G$ is a $p$-group. Suppose that $G$ is not cyclic. It suffices to prove that $G \in \Phi$. Since the center of a $p$-group is non-trivial, we may assume that $\langle a\rangle$ is a cyclic subgroup of order $p$ and is contained in the center of $G$. If $G$ is a group of exponent $p$, that is, every element of $G$ has order $p$, then clearly, $G$ satisfies (iii), as desired. As a result, we may take a maximal cyclic subgroup $\langle b\rangle$ of order at least $p^{2}$. Suppose for a contradiction that $a \notin\langle b\rangle$. Then $\langle a\rangle\langle b\rangle=\langle a, b\rangle$ is an abelian subgroup of $G$. It follows that $\langle b\rangle$ is normal in $\langle a\rangle\langle b\rangle$. Since $\langle a\rangle$ is normal in $\langle a\rangle\langle b\rangle$ and $\langle a\rangle \cap\langle b\rangle=\{e\}$, we have

$$
\langle a\rangle\langle b\rangle=\langle a\rangle \times\langle b\rangle \cong \mathbb{Z}_{p} \times \mathbb{Z}_{p^{m}},
$$

where $|\langle b\rangle|=p^{m}$ for some $m \geqslant 2$. It follows that $G$ has a subgroup isomorphic to $\mathbb{Z}_{p} \times \mathbb{Z}_{p^{2}}$, contrary to Lemma 2 (i). We conclude $a \in\langle b\rangle$.

Suppose for a contradiction that $G$ has a maximal cyclic subgroup $\langle c\rangle$ of order at least $p^{2}$ with $\langle c\rangle \neq\langle b\rangle$. Similarly, we have $a \in\langle c\rangle$. It follows from Observation 4 that one of $\langle c\rangle \subseteq\langle b\rangle$ and $\langle b\rangle \subseteq\langle c\rangle$ occurs. Hence, we have $\langle c\rangle=\langle b\rangle$ since both $\langle b\rangle$ and $\langle c\rangle$ are maximal cyclic, a contradiction. We conclude that $G \in \Phi$, as desired.

In view of Lemma 2 , the next result is obtained by applying Theorem 5 to abelian groups.

Corollary 5 Let $G$ be an abelian group. Then $\mathscr{P}_{I}(G)=\mathscr{P}(G)$ if and only if $G$ is isomorphic to one of $\mathbb{Z}_{p^{m}}, \mathbb{Z}_{p}^{m}$, and $\mathbb{Z}_{p q}$, where $p, q$ are distinct primes and $m$ is a positive integer.

By (2)-(6), we have the following result.

\section{Corollary 6}

(i) Let $n \geqslant 3$. Then $\mathscr{P}_{I}\left(D_{2 n}\right)=\mathscr{P}\left(D_{2 n}\right)$ if and only if $n$ is either a prime power or a product of two distinct primes.

(ii) Let $m \geqslant 2$. Then $\mathscr{P}_{I}\left(Q_{4 m}\right) \neq \mathscr{P}\left(Q_{4 m}\right)$.

\section{Intersection power graph and enhanced power graph}

A group is called a CP-group [20] if every element of the group has prime power order. For example, every $p$-group is a CP-group. 
Theorem 6 The following conditions are equivalent for a group $G$ :

(a) $\mathscr{P}_{I}(G)=\mathscr{P}_{E}(G)$;

(b) For any $x, y \in G \backslash\{e\},\langle x\rangle \cap\langle y\rangle \neq\{e\}$ is equivalent to $\langle x, y\rangle$ is cyclic;

(c) $\mathscr{P}^{*}(G)$ is a disjoint union of some complete graphs, that is, every connected component of $\mathscr{P}^{*}(G)$ is complete;

(d) $G$ is a CP-group in which every two distinct maximal cyclic subgroups has trivial intersection.

Proof: By the definitions of the intersection power graph and the enhanced power graph, it is clear that (a) and (b) are equivalent.

In the following, we prove (b) implies (d). Suppose that (b) holds. Assume to the contrary, that $G$ has an element $x$ of order $p q$ where $p$ and $q$ are distinct primes. Then $o\left(x^{p}\right)=q, o\left(x^{q}\right)=p$, and $\left\langle x^{p}, x^{q}\right\rangle \subseteq\langle x\rangle$ is cyclic. Thus, by (b) we have $\left\langle x^{p}\right\rangle \cap\left\langle x^{q}\right\rangle \neq\{e\}$, a contradiction. It follows that every element of $G$ has prime power order, and so $G$ is a CP-group. We next prove that any two distinct maximal cyclic subgroups of $G$ have trivial intersection. Otherwise, let $\left\langle x_{1}\right\rangle$ and $\left\langle x_{2}\right\rangle$ be distinct maximal cyclic subgroups of $G$ such that $\mid\left\langle x_{1}\right\rangle \cap$ $\left\langle x_{2}\right\rangle \mid>1$. Then both $o\left(x_{1}\right)$ and $o\left(x_{2}\right)$ are powers of a prime, say, $p$. It follows from (b) that $\left\langle x_{1}, x_{2}\right\rangle$ is cyclic. Since both $\left\langle x_{1}\right\rangle$ and $\left\langle x_{2}\right\rangle$ are maximal cyclic, we have $\left\langle x_{1}, x_{2}\right\rangle=\left\langle x_{1}\right\rangle=\left\langle x_{2}\right\rangle$, a contradiction as $\left\langle x_{1}\right\rangle \neq\left\langle x_{2}\right\rangle$. We conclude that (d) follows.

Now we prove (d) implies (c). Suppose that (d) holds. Let $\mathscr{M}_{G}=\left\{P_{1}, P_{2}, \ldots, P_{t}\right\}$ for some positive integer $t$. Note that for any $1 \leqslant i \leqslant t,\left|P_{i}\right|$ is a prime power. By Lemma 3, the subgraph $\mathscr{P}^{*}\left(P_{i}\right)$ of $\mathscr{P} *(G)$ induced by $P_{i} \backslash\{e\}$ is complete. Also, since $\left|P_{i} \cap P_{j}\right|=1$ for all $1 \leqslant j \leqslant t, j \neq i$, we have that $\mathscr{P}^{*}(G)$ is a disjoint union of the complete graphs $\mathscr{P}^{*}\left(P_{1}\right), \mathscr{P}^{*}\left(P_{2}\right), \ldots, \mathscr{P}^{*}\left(P_{t}\right)$, which proves (c).

Finally, we prove (c) implies (b). Suppose that (c) is valid. Take $x, y \in G \backslash\{e\}$. Suppose first that $\langle x, y\rangle=\langle z\rangle$. In the following we show $\langle x\rangle \cap\langle y\rangle \neq$ $\{e\}$. Let $\Gamma$ be a connected component of $\mathscr{P}^{*}(G)$ which contains $z$ as a vertex. It follows that $x, y \in$ $V(\Gamma)$, since $z$ is adjacent to every other element of $\langle z\rangle$ in $\mathscr{P}(G)$. Notice that an element of order $q$ and an element of order $r$ are non-adjacent in $\mathscr{P}(G)$, where $q \neq r$ are primes. Since $\Gamma$ is complete, we have that $o(z)$ is a power of a prime, say $p$. This also implies that both $o(x)$ and $o(y)$ are powers of $p$. As a result, $\langle x\rangle \subseteq\langle y\rangle$ or $\langle y\rangle \subseteq\langle x\rangle$, namely, $\langle x\rangle \cap\langle y\rangle \neq\{e\}$, as desired.
Suppose now $\langle x\rangle \cap\langle y\rangle \neq\{e\}$. It suffices to prove that $\langle x, y\rangle$ is cyclic. Let $w \in\langle x\rangle \cap\langle y\rangle$ such that $o(w)=p$ for some prime $p$. Thus, if $w \neq x$ (resp., $w \neq y$ ), then $w$ is adjacent to $x$ (resp., $y$ ) in $\mathscr{P}(G)$. As a result, $x, y$ belong to a connected component of $\mathscr{P}^{*}(G)$, say, $\Delta$. Since $\Delta$ is complete, we have $\langle x\rangle \subseteq\langle y\rangle$ or $\langle y\rangle \subseteq\langle x\rangle$, and so $\langle x, y\rangle=\langle x\rangle$ or $\langle y\rangle$, that is, $\langle x, y\rangle$ is cyclic, as desire.

Lemma 4 (Theorem 28 [11]) For a finite group $G$, $\mathscr{P}_{E}(G)=\mathscr{P}(G)$ if and only if every cyclic subgroup of $G$ has prime power order.

Combining Theorems 5 and 6, and Lemma 4, we have the following corollary.

Corollary 7 For a nilpotent group $G, \mathscr{P}_{I}(G)=$ $\mathscr{P}_{E}(G)$ if and only if either $G$ is a cyclic p-group or $G \in \Phi$.

Remark that if $G$ is an abelian $p$-group and has exponent at least $p^{2}$, then $G$ has a subgroup isomorphic to $\mathbb{Z}_{p} \times \mathbb{Z}_{p^{2}}$, and so $G \notin \Phi$. It follows that $G \in \Phi$ is an abelian p-group if and only if $G$ has exponent $p$. Now by Corollary 7 and (2)-(6), one can easily obtain following result.

\section{Corollary 8}

(i) Let $G$ be an abelian group. Then $\mathscr{P}_{I}(G)=\mathscr{P}_{E}(G)$ if and only if $G$ is either a cyclic p-group or an elementary abelian p-group.

(ii) Let $n \geqslant 3$. Then $\mathscr{P}_{I}\left(D_{2 n}\right)=\mathscr{P}_{E}\left(D_{2 n}\right)$ if and only if $n$ is a prime power.

(iii) Let $m \geqslant 2$. Then $\mathscr{P}_{I}\left(Q_{4 m}\right) \neq \mathscr{P}_{E}\left(Q_{4 m}\right)$.

\section{Intersection power graph and commuting graph}

Notice that $\mathscr{C}(G)$ is complete if and only if $G$ is abelian. The following observation follows from the definitions of intersection power graph and commuting graph.

Observation 7 For a group $G$, the intersection power graph of $G$ is equal to its commuting graph if and only if $G$ satisfies the property: for any $x, y \in G \backslash\{e\},\langle x\rangle \cap$ $\langle y\rangle \neq\{e\}$ is equivalent to $x y=y x$.

Theorem 8 For a group $G, \mathscr{P}_{I}(G)=\mathscr{C}(G)$ if and only if $G$ satisfies the following:

(i) $G$ is a CP-group;

(ii) $G$ has no subgroup $\mathbb{Z}_{p} \times \mathbb{Z}_{p}$ for a prime $p$;

(iii) Every two distinct maximal cyclic subgroups of $G$ has trivial intersection.

Proof: We first prove the necessity. Suppose that $\mathscr{P}_{I}(G)=\mathscr{C}(G)$. If $G$ has an element $a$ of order $p q$ 
where $p, q$ are distinct primes, then $a^{p} a^{q}=a^{q} a^{p}$ and $\left\langle a^{p}\right\rangle \cap\left\langle a^{q}\right\rangle=\{e\}$, contrary to Observation 7 . This means that $G$ is a CP-group, and so (i) follows. Now suppose for a contradiction that $\mathbb{Z}_{p} \times \mathbb{Z}_{p}$ is a subgroup of $G$, where $p$ is a prime. Then $G$ has at least two distinct cyclic subgroups of order $p$ in $\mathbb{Z}_{p} \times \mathbb{Z}_{p}$, say, $\langle x\rangle$ and $\langle y\rangle$. Clearly, $x y=y x$ and $\langle x\rangle \cap\langle y\rangle=\{e\}$, this contradicts Observation 7. We conclude that (ii) is valid. It now suffices to show (iii).

Suppose for a contradiction that there exist two distinct maximal cyclic subgroups, say, $\langle x\rangle,\langle y\rangle$, of $G$ such that $\langle x\rangle \cap\langle y\rangle \neq\{e\}$. Since is a CP-group, we have that both $o(x)$ and $o(x)$ are powers of some prime, say, $p$. Now Observation 7 implies $x y=y x$. It follows that $\langle x, y\rangle$ is abelian, and hence is a direct product of some cyclic groups, here every cyclic group has order $p^{m}$ for some $m \geqslant 1$. Notice that $G$ has no subgroup $\mathbb{Z}_{p} \times \mathbb{Z}_{p}$. It follows that $\langle x, y\rangle$ is a cyclic $p$-group, and so $\langle x, y\rangle=\langle x\rangle=\langle y\rangle$, a contradiction. We conclude that (iii) follows.

We next prove the sufficiency. Suppose that $G$ satisfies the three conditions (i)-(iii). Take $x, y \in$ $G \backslash\{e\}$ such that $\langle x\rangle \cap\langle y\rangle \neq\{e\}$. Then by (i), both $o(x)$ and $o(y)$ are powers of some prime, say, $p$. Also, by (iii), it follows that both $\langle x\rangle$ and $\langle x\rangle$ must be contained in the same maximal cyclic subgroup of $G$, which implies $x y=y x$. On the other hand, suppose that $a b=b a$ for two distinct $a, b \in G \backslash\{e\}$. In view of Observation 7, it suffices to prove $\langle a\rangle \cap\langle b\rangle \neq\{e\}$. Notice that $\langle x, y\rangle$ is abelian. We have that $\langle x, y\rangle$ is a direct product of two cyclic groups, say, $\mathbb{Z}_{m} \times \mathbb{Z}_{n}$. Considering the condition (ii), we have that $m$ and $n$ are coprime. By (i) again, it follows that $\langle x, y\rangle$ is a cyclic group of prime power order. Without loss of generality, now assume that $o(x) \leqslant o(y)$. We conclude that $\langle x\rangle \cap\langle y\rangle=\langle x\rangle$, as desired.

Corollary 9 For a nilpotent group $G, \mathscr{P}_{I}(G)=\mathscr{C}(G)$ if and only if $G$ is a cyclic p-group.

Proof: If $G$ is a cyclic p-group, then Corollary 1 implies that $\mathscr{P}_{I}(G)$ is complete, and so $\mathscr{P}_{I}(G)=$ $\mathscr{C}(G)$, as desired. Now suppose that $\mathscr{P}_{I}(G)=\mathscr{C}(G)$. By Theorem $8, G$ is a CP-group. As a result, $G$ is a $p$-group for some prime $p$ since $G$ is nilpotent. Since the center of a $p$-group is non-trivial, we may take a subgroup of order $p$ which is included in the center of $G$, say, $\langle x\rangle$. If $G$ has another subgroup $\langle y\rangle$ of order $p$, then $\langle x, y\rangle \cong \mathbb{Z}_{p} \times \mathbb{Z}_{p}$, contrary to Theorem 8 . We conclude that $G$ has a unique subgroup of order $p$. It follows from Lemma 1 that $G$ is either cyclic or generalized quaternion group. If $G$ is a generalized quaternion 2-group, then (6) implies that there exist two distinct maximal cyclic subgroups in $G$ such that their intersection has size 2 , which is impossible by Theorem 8. Consequently, we have that $G$ is a cyclic $p$-group, as required.

An element of order 2 in $G$ is called an involution.

\section{Corollary 10}

(i) Let $n \geqslant 3$. Then $\mathscr{P}_{I}\left(D_{2 n}\right)=\mathscr{C}\left(D_{2 n}\right)$ if and only if $n$ is a power of some odd prime.

(ii) Let $m \geqslant 2$. Then $\mathscr{P}_{I}\left(Q_{4 m}\right) \neq \mathscr{C}\left(Q_{4 m}\right)$.

Proof: (i) Suppose that $\mathscr{P}_{I}\left(D_{2 n}\right)=\mathscr{C}\left(D_{2 n}\right)$. By Theorem $8, D_{2 n}$ is a CP-group, so $n$ is a prime power. If $n$ is even, then by (4), $Z\left(D_{2 n}\right)$ has an involution, and since $D_{2 n}$ has precisely $n+1$ involutions by (2), we have that $D_{2 n}$ has a subgroup isomorphic to $\mathbb{Z}_{2} \times \mathbb{Z}_{2}$, contrary to Theorem 8 . It follows that $n$ is a power of some odd prime, as desired. On the other hand, by (2) and (3), it is easy to see that if $n$ is a power of some odd prime, then $\mathscr{P}_{I}\left(D_{2 n}\right)=\mathscr{C}\left(D_{2 n}\right)$.

(ii) The result follows from Theorem $8,(5)$, and (6).

\section{Intersection power graph and order supergraph}

In this subsection, we classify completely the groups whose intersection power graphs are equal to their order supergraphs.

Lemma 5 Let $G$ be a group satisfying $\mathscr{P}_{I}(G)=$ $\mathscr{S}(G)$. Then

(i) $G$ has no element of order pqr or $p^{2} q$, where $p, q, r$ are pairwise distinct primes;

(ii) If $p$ is a prime dividing $|G|$, then $G$ has exactly one subgroup of order $p$.

Proof: (i) Suppose for a contradiction that $G$ has an element $x$ of order $p q r$, where $p, q, r$ are pairwise distinct primes. Then $x^{p}$ is adjacent to $x^{q}$ in $\mathscr{P}_{I}(G)$ as $x^{p q} \in\left\langle x^{p}\right\rangle \cap\left\langle x^{q}\right\rangle$. However, $x^{p}$ and $x^{q}$ are nonadjacent in $\mathscr{S}(G)$ as $o\left(x^{p}\right)=q r$ and $o\left(x^{q}\right)=p r$, contrary to $\mathscr{P}_{I}(G)=\mathscr{S}(G)$. Similarly, we also can obtain that $G$ has no element of order $p^{2} q$, where $p, q$ are distinct primes.

(ii) Suppose for a contradiction that $G$ has two distinct subgroups of order $p$, say, $\langle x\rangle$ and $\langle y\rangle$. Then $x$ is adjacent to $y$ in $\mathscr{S}(G)$, but $x$ is not adjacent to $y$ in $\mathscr{P}_{I}(G)$ as $\langle x\rangle \cap\langle y\rangle=\{e\}$, a contradiction.

Theorem 9 For a group $G$, the intersection power graph of $G$ is equal to its order supergraph if and only if $G$ is isomorphic to one of the following groups:

(a) $\mathbb{Z}_{p q}$, where $p$ and $q$ are distinct primes;

(b) $\mathbb{Z}_{p^{n}}$, where $p$ is a prime and $n$ is a positive integer; 
(c) $Q_{4 \cdot 2^{k}}$, where $k$ is a positive integer.

Proof: Clearly, $\mathscr{P}_{I}\left(\mathbb{Z}_{p q}\right)=\mathscr{S}\left(\mathbb{Z}_{p q}\right)$ for distinct primes $p, q$. Also, Corollary 1 implies that if $G$ is either a cyclic $p$-group or a generalized quaternion 2-group, then $\mathscr{P}_{I}(G)$ is complete. Furthermore, it is clear that if $G$ is a $p$-group, then $\mathscr{S}(G)$ is complete. Therefore, the sufficiency follows. In the following, we prove the necessity.

Suppose that $\mathscr{P}_{I}(G)=\mathscr{S}(G)$. Assume to the contrary that $|G|$ has three pairwise distinct prime divisors, say, $p, q, r$. Let $\langle x\rangle,\langle y\rangle,\langle z\rangle$ be three subgroups of order $p, q, r$, respectively. By Lemma 5 (ii), we have that every of $\langle x\rangle,\langle y\rangle,\langle z\rangle$ is normal in $G$. It follows that $\langle x, y, z\rangle \cong \mathbb{Z}_{p q r}$, contrary to Lemma 5 (i). We conclude that $|G|$ has at most two prime divisors.

Case 1: $|G|=p^{m} q^{n}$, where $p, q$ are distinct primes and $m, n$ are positive integers.

Let $\langle x\rangle,\langle y\rangle$ be two subgroups of order $p, q$, respectively. Then Lemma 5 (ii) implies that $\langle x\rangle$ and $\langle y\rangle$ are unique subgroups of order $p, q$ in $G$, respectively. Therefore, $\langle x\rangle$ and $\langle y\rangle$ are normal subgroups, which implies that $x$ and $y$ commute. It follows that $x y$ has order $p q$ and $x, y \in\langle x y\rangle$. We now claim that $G$ has no element of order $p^{2}$ or $q^{2}$. In fact, if $G$ has an element of order $p^{2}$, say, $a$, then $x \in\langle a\rangle$, and so $x \in\langle a\rangle \cap\langle x y\rangle$, which is a contradiction since $o(x y)=p q$ and $o(a)=p^{2}$. Similarly, we also have that $G$ has no element of order $q^{2}$. Thus, our claim is valid. Now let $P$ and $Q$ are Sylow $p$-subgroup and Sylow $q$-subgroup of $G$, respectively. Then $P$ and $Q$ are elementary abelian $p$-group and elementary abelian $q$-group, respectively. In view of Lemma 5 (ii), we have that $P \cong \mathbb{Z}_{p}$ and $Q \cong \mathbb{Z}_{q}$. As a result, $G \cong \mathbb{Z}_{p q}$, as desired.

Case 2. $|G|=p^{m}$, where $p$ is a prime and $m$ is a positive integer.

Combining Lemma 5 (ii) and Lemma 1, we have that $G$ is either a cyclic group or a generalized quaternion 2-group, as desired.

Acknowledgements: The second author was supported by the National Natural Science Foundation of China (Grant No. 11801441), the Natural Science Basic Research Program of Shaanxi (Program No. 2020JQ-761), and the Young Talent fund of University Association for Science and Technology in Shaanxi, China (Program No. 20190507).

\section{REFERENCES}

1. Kelarev AV, Quinn SJ (2000) A combinatorial property and power graphs of groups. Contrib General Algebra 12, 229-235.
2. Chakrabarty I, Ghosh S, Sen MK (2009) Undirected power graphs of semigroups. Semigroup Forum 78, 410-426.

3. Kelarev AV, Quinn SJ (2002) Directed graphs and combinatorial properties of semigroups. $J$ Algebra 251, 16-26.

4. Kelarev AV, Quinn SJ (2004) A combinatorial property and power graphs of semigroups. Comment Math Uni Carolinae 45, 1-7.

5. Kelarev AV, Quinn SJ, Smolikova R (2001) Power graphs and semigroups of matrices. Bull Austral Math Soc 63, 341-344.

6. Doostabadi A, Erfanian A, Jafarzadeh A (2015) Some results on the power graphs of finite groups. ScienceAsia 41, 73-78.

7. Abawajy J, Kelarev A, Chowdhury M (2013) Power graphs: A survey. Electron J Graph Theory Appl 1, 125-147.

8. Brauer R, Fowler KA (1955) On groups of even order. Ann Math 62, 567-583.

9. Araújo J, Bentz W, Janusz K (2015) The commuting graph of the symmetric inverse semigroup. Isr J Math 207, 103-149.

10. Giudici M, Pope A (2014) On bounding the diameter of the commuting graph of a group. $J$ Group Theory 17, 131-149.

11. Aalipour G, Akbari S, Cameron PJ, Nikandish R, Shaveisi F (2017) On the structure of the power graph and the enhanced power graph of a group. Electron J Combin 24, ID P3.16.

12. Bera S, Bhuniya AK (2018) On enhanced power graphs of finite groups. J Algebra Appl 17, ID 1850146.

13. Hamzeh A, Ashrafi AR (2017) Automorphism group of supergraphs of the power graph of a finite group. European J Combin 60, 82-88.

14. Hamzeh A, Ashrafi AR (2018) The order supergraph of the power graph of a finite group. Turk J Math 42, 1978-1989.

15. Bera S (2018) On the intersection power graph of a finite group. Electron J Graph Theory Appl 6, 178-189.

16. Aprose MA, Fathima SSA (2020) Further results on intersection power graphs of finite groups. Punjab Univ J Math 52, 47-53.

17. Johnson DL (1980) Topics in the Theory of Group Presentations, London Math Soc Lecture Note Ser 42, Cambridge University Press, UK.

18. Gorenstein D (1980) Finite Groups, Chelsea Publishing Co, New York.

19. Deaconescu M (1989) Classification of finite groups with all elements of prime order. Proc Amer Math Soc 106, 625-629.

20. Higman G (1957) Finite groups in which every element has prime power order. $J$ London Math Soc 32, 335-342. 